\title{
BMJ Open Breastfeeding peer counselling for mothers of preterm neonates: protocol of a stepped-wedge cluster randomised controlled trial
}

\author{
Sophie Laborie (D) , ${ }^{1}$ Angelique Denis, ${ }^{2,3}$ Antje Horsch, ${ }^{4,5}$ Pauline Occelli, ${ }^{6,7}$ \\ Jennifer Margier (D) , 8 Mathilde Morisod Harari, ${ }^{9}$ Olivier Claris, ${ }^{10,11}$ \\ Sandrine Touzet, ${ }^{12,13}$ Celine Julie Fischer Fumeaux ${ }^{5}$
}

To cite: Laborie S, Denis A, Horsch A, et al. Breastfeeding peer counselling for mothers of preterm neonates: protocol of a steppedwedge cluster randomised controlled trial. BMJ Open 2020;10:e032910. doi:10.1136/ bmjopen-2019-032910

- Prepublication history for this paper is available online. To view these files, please visit the journal online (http://dx.doi. org/10.1136/bmjopen-2019032910).

Received 18 July 2019 Revised 03 December 2019 Accepted 19 December 2019

Check for updates

(C) Author(s) (or their employer(s)) 2020. Re-use permitted under CC BY-NC. No commercial re-use. See rights and permissions. Published by BMJ.

For numbered affiliations see end of article.

Correspondence to

Dr Sophie Laborie;

sophie.laborie@chu-lyon.fr

\section{ABSTRACT}

Introduction Among preterm infants, mother's own milk feeding reduces neonatal morbidity and decreases the length of hospital stay. However, breastfeeding rates and duration are lower than among term infants. It is reported that peer counselling is effective in increasing breast feeding in term infants in low-income and middle-income countries, but results are mixed in high-income countries. We aim to investigate herein whether peer counselling may be a feasible and effective breastfeeding support among preterm infants in French-speaking high-income countries.

Methods and analysis Eight European centres will participate in this stepped-wedge cluster randomised controlled trial. We plan to include 2400 hospitalised neonates born before 35 gestational weeks. Each centre will begin with an observational period. Every 3 months, a randomised cluster (centre) will begin the interventional period with peer counsellors until the end of the study. The counsellors will be trained and supervised by the trained nurses. They will have a weekly contact with participating mothers, with a face-to-face meeting at least once every fortnight. During these meetings, peer counsellors will listen to mothers' concerns, share experiences and help the mother with their own knowledge of breast feeding. The main outcome is breastfeeding rate at 2 months corrected age. Secondary outcomes are breastfeeding rates at hospital discharge and at 6 months, breastfeeding duration and severe neonatal morbidity and mortality. The mental health of the mother, mother-infant bonding and infant behaviour will be assessed using self-report questionnaires. A neurodevelopmental follow-up, a costeffectiveness analysis and a cost-consequence at 2 years corrected age will be performed among infants in a French subgroup.

Ethics and dissemination French, Belgian and Swiss ethics committees gave their agreement. Publications in peer-reviewed journals are planned on breast feeding, mental health and economic outcomes.

Trial registration number NCT03156946

\section{INTRODUCTION}

According to a recent meta-analysis, increasing breastfeeding rates following the
Strengths and limitations of this study

- This study is the first multicentre and multinational randomised trial investigating the efficacy of peer counsellors to support and improve breast feeding of preterm infants.

- This study gives us the opportunity to test the feasibility of such an intervention in Europe.

- The potential effect of peer counselling on maternal mental health and mother-infant bonding will also be investigated.

- The cost-consequence analysis and the costeffectiveness ratio expressed as the cost by avoided impairment at 2 years corrected age will be calculated for a subgroup of infants from a French geographical region.

- The measurement of psychological outcomes is limited by the use of self-report questionnaires.

WHO recommendations could prevent yearly more than 800000 deaths of children below the age of 5 years $^{1}$ and could save more than 300 billion dollars per year. ${ }^{2}$

Prematurity represents the leading cause of infant mortality around the world, and can lead to severe short-term and long-term complications. In preterm neonates, mother's own milk feeding is associated with a significant decrease of severe morbidities, such as sepsis, ${ }^{3-6}$ enterocolitis, ${ }^{367}$ retinopathy of prematurity ${ }^{8}$ and bronchopulmonary dysplasia. ${ }^{9}$ It has been shown to reduce the duration of hospital stay, as well as the risk of rehospitalisation. ${ }^{1011}$ In the same population, it is also associated with an increase of neurodevelopmental or cognitive scores in infancy and childhood ${ }^{12-15}$ with a dose-dependent effect. ${ }^{12}{ }^{16}$ However, studies have shown that breastfeeding rates of preterm infants at hospital discharge are far below those of term infants. ${ }^{17}$ 
In term infants, peer counselling by mothers with a previous positive experience of breast feeding is effective in promoting breast feeding in low-income and middle-income countries (relative risk $0.70,95 \%$ CI 0.60 to 0.82$)) .{ }^{18-21}$ In high-income countries results are mixed. ${ }^{182122}$

In neonatal intensive care units (NICUs), support by 'veteran' parents with previous comparable experiences supporting NICU parents can have important benefits in term of psychological health for the parents, such as reduced stress, anxiety and depression, and increased perceived social support. ${ }^{23}$ The studies on breastfeeding peer support in NICUs are scarce. An American randomised trial showed a significant increase in breast feeding at 12 weeks post partum in the group with peer counsellors $(\mathrm{n}=85$, OR $2.81,95 \%$ CI 1.1 to 7.14$){ }^{24}$ Furthermore, the beneficial effect of peer support in NICUs increased even further by the cointervention of a breastfeeding consultant, ${ }^{25}$ as already demonstrated for full-term infants. ${ }^{26}$

This multicentre randomised cluster trial aims to study the effect of breastfeeding peer counselling for mothers of preterm neonates. The primary objective is to assess breastfeeding rates among preterm neonates at 2 months corrected age. The secondary objectives are to assess breastfeeding rates at hospital discharge and at 6 months, breastfeeding duration and neonatal mortality and severe morbidity. Furthermore, psychological consequences of the intervention on maternal mental health, mother-infant bonding and infant behaviour will be investigated. Moreover, we aim to assess satisfaction with the peer breastfeeding support and to measure its implementation. A neurodevelopmental follow-up and a cost-effectiveness analysis at 2 years corrected age will be performed. Finally, the feasibility of such a peer support system for preterm breastfeeding support in European countries will be assessed.

\section{METHODS}

\section{Design and setting}

The study is a stepped-wedge cluster randomised controlled trial. The design is presented in figure 1. Each NICU corresponds to a cluster. All clusters start in the control situation at the beginning of the study. At each time step (every third month), a new cluster will crossover from the control period to the transition period and 3 months later to the intervention period. This design was chosen to prevent contamination of the intervention in the centre and to allow the delivery of the intervention in all participating centres.

The order of implementation is randomised, based on a computer-generated random sequence performed by an independent researcher. Due to the nature of the intervention, it is not possible to blind mothers and teams in NICUs.

During the transition period, the centres do not contribute to analyses. This transition period takes into account the time it takes for the recruitment and training of peer counsellors (figure 1).

The trial is conducted in eight NICUs, six of which are in France, one is in Switzerland and one in Belgium. One of the French centres is located in the Overseas Territories. The inclusions have started on 5 November 2018.

In each centre, two specialist lactation nurses or lactation consultants will supervise the peer support intervention. All supervisors participate in an identical 5-day training course, enabling them to recruit, train and supervise counsellors. This training is provided twice (in October 2018 for the first four centres and in November 2019 for the others) by an organisation (Association Relai Parentalite Allaitement) that has experience in training supervisors for peer support networks, including NICU networks.

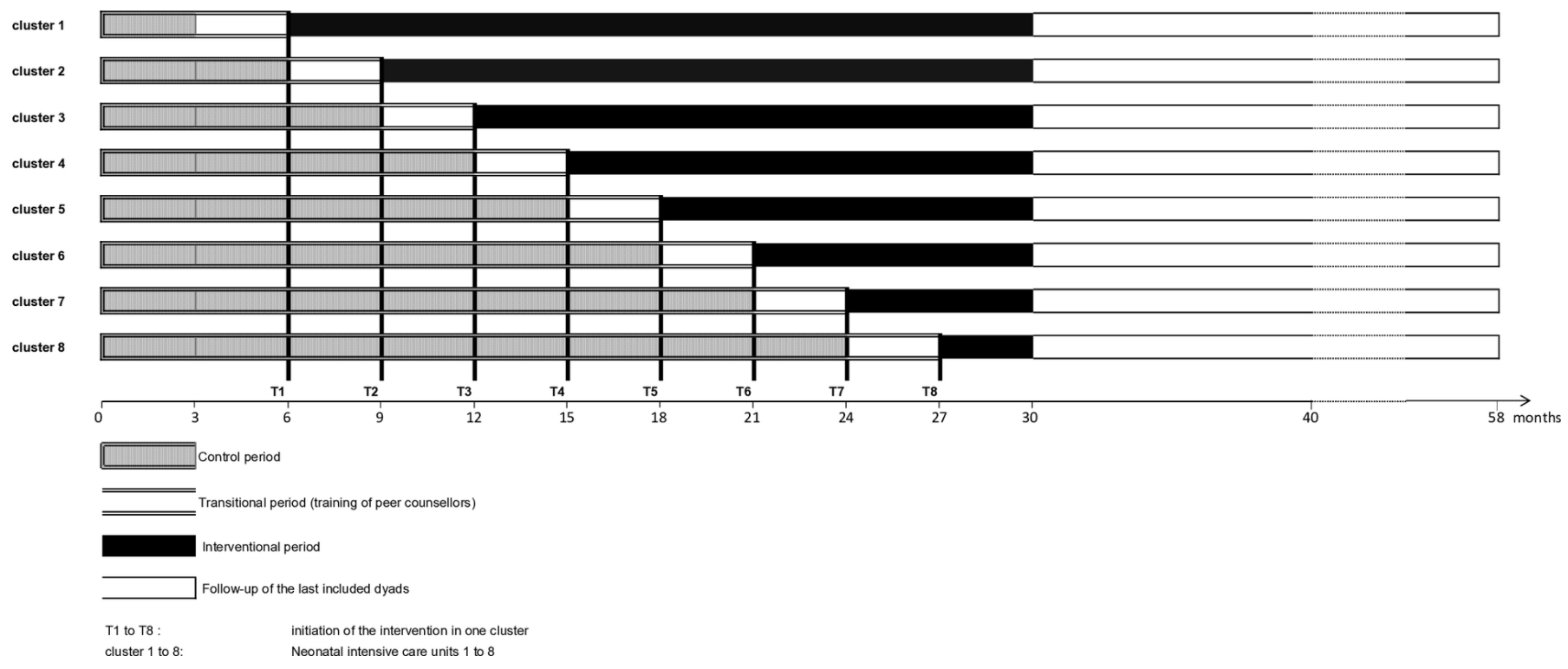

Figure 1 Study design. 
Inclusion and exclusion criteria

The mother and child dyads can be included if:

- Mothers:

Deliver before 35 weeks of gestational age.

Do not present a medical contraindication to breast feeding.

Have sufficient language (French) skills to adequately participate in the study.

Give their informed consent during the 7 days following delivery.

- Neonates:

Are admitted to a neonatal unit in the first 24 hours following birth.

Do not present a medical contraindication to breast feeding.

Serious mental disorder in mothers and life threatening congenital malformations in infants constitute exclusion criteria.

\section{Peer counsellors}

Peers counsellors are eligible if they have breast fed at least one preterm infant hospitalised in an NICU, if they had a positive experience of breast feeding, and if the breastfed child is in good health. In each NICU, all mothers of living former preterm babies aged between 1 and 3 years are invited to an information meeting. Thereafter the volunteers are interviewed and selected by the supervisors and participate in a 20-hour training programme led by the supervisors. The aim of the training is to provide them with knowledge about frequent difficulties encountered in breast feeding of a preterm infant and to train them through role-plays to engage in a helping relationship. ${ }^{27}$ They are not paid, although they will be partially compensated for travel or telephone costs. The number of peers selected depends on the size of each centre and varies from 9 to 29, taking into account an attrition rate of $30 \%$ per year. If needed, a new selection and training can be organised during the study.

\section{Control period}

The mothers will receive the professional breastfeeding support available (usual care). The organisation of this support depends on NICUs with various combinations and levels of support by nurses and/or breastfeeding consultants. In the Swiss centre, a collective peer support with a weekly meeting was already in place before the study: it will continue throughout the study.

After discharge, the mothers are supported by paediatric nurses and, in Switzerland and in Belgium, by the independent midwives.

The difference between usual care will be partly recorded in the case report form (CRF) (intent of breast feeding, sources of information on breast feeding during the pregnancy, interval between birth and first use of breast pump or manual expression of milk, type of breastfeeding support received, number of skin-to-skin contacts during the first week, raw maternal milk administration and the date of first administration, date of first oral feeding and its modality, and duration of stay in a kangaroo/mother unit). It will also be evaluated for each centre through a questionnaire, ${ }^{28}$ given to each unit at the initiation of the study, during the transition period and at the end of the study.

\section{Intervention period}

In addition to existing professional lactation support, mothers will receive an individual peer support by one of the peer counsellors.

Peer counsellors will first visit mothers either in the obstetrical unit before birth in case of premature delivery risk or in the NICU. Then, during the NICU stay, at least weekly contact between peer counsellors and participating mothers (face to face, videotelephony or telephone) will be planned, with a face-to-face meeting at least once every fortnight.

After infant discharge from the NICU, or if the infant is transferred to another hospital, the weekly contact will continue by phone until 1 month after the infant's return to the parental home. The minimum total number of contacts is five. The duration of the meetings is on average between three-quarter and 1 hour.

During these meetings, peer counsellors (matched for having had a child of comparable weight) will listen to mothers' concerns, share experiences on prematurity or breast feeding and help the mother with their own knowledge of breast feeding. If they encounter difficulties, they can contact the supervisors individually or discuss them during a monthly supervisory group meeting. They record their interventions (dates, type and topics).

The peer breastfeeding support is planned to stop in case of weaning or 1 month after hospital discharge, whichever occurs first. If the mother or the counsellor asks for discontinuation, another counsellor will be proposed to participating mothers.

Premature discontinuation of the intervention may happen in case of infant or maternal death, of withdrawal of consent, or if the mother asks for it and refuses another counsellor. In such cases, infants will be followed up until the end of the study period, except if the parents refuse this.

\section{Outcomes and measures}

- Primary outcome and measure

The primary outcome is breastfeeding rate at 2 months corrected age. An infant who received the mother's own milk during the preceding 48 hours will be considered as breast fed. The infant feeding will be assessed through telephone calls.

- Secondary outcomes and measures All measures and their timings are listed in table 1.

\section{Breast feeding}

Breastfeeding initiation is defined as receiving at least once the mother's own milk. Breast feeding at discharge is defined as receiving some mother's own milk during the 48 hours before NICU discharge. Breast feeding at 6 


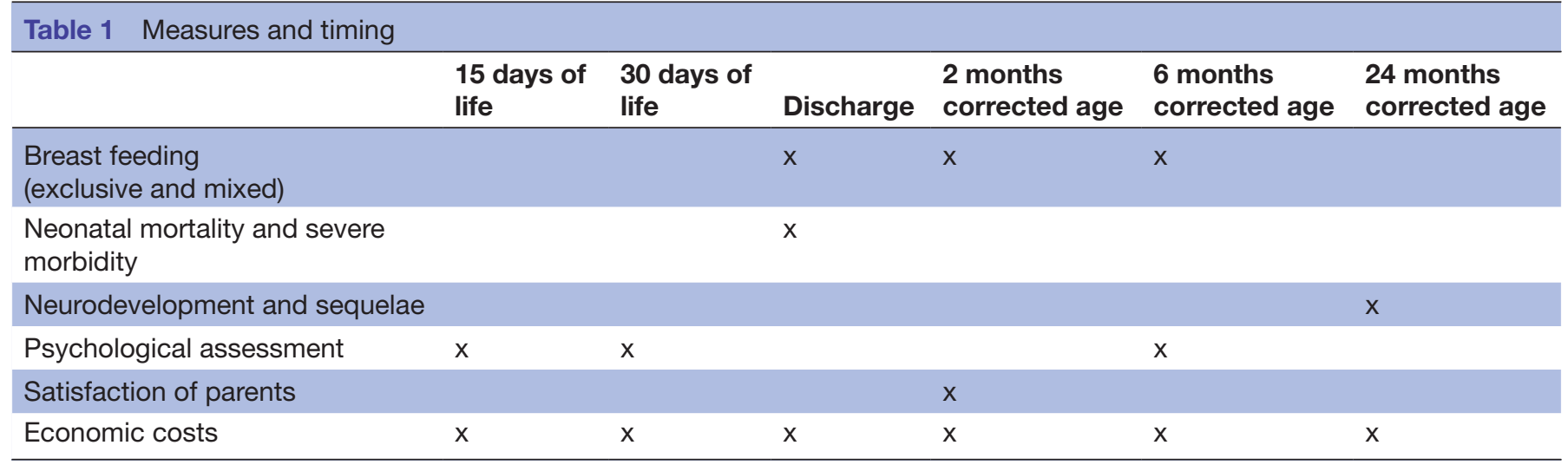

months corrected age is defined as receiving any mother's own milk in the 48 hours before 6 months corrected age. Exclusive breast feeding is defined as receiving neither other milk nor food than their mother's own milk during the 48 hours before discharge, and 2 and 6 months corrected age. Duration of breast feeding is defined as duration from birth until last administration of mother's own milk. If breast feeding is continuing at 6 months corrected age, the longer duration will not be recorded (censored data).

\section{Neonatal mortality and severe morbidities}

Mortality and the following complications (intraventricular haemorrhage (grade $>2),{ }^{29}$ periventricular leukomalacia, enterocolitis stage $>1,30$ bronchopulmonary dysplasia defined as a persistent oxygen dependency or respiratory support at 36 weeks corrected age, ${ }^{31}$ persistent ductus arteriosus requiring treatment, retinopathy of prematurity grade $>2,{ }^{32}$ sepsis (proven or probable) ${ }^{33}$ will be assessed during the hospitalisation until 36 weeks corrected age.

\section{Neurodevelopmental outcomes}

In infants born before 33 weeks gestational age or with a weight below $1500 \mathrm{~g}$ living in a specific geographical French area, the neurodevelopment will be assessed at 24 months using the Brunet-Lézine scale. ${ }^{34}$ The BrunetLézine scale measures four different subscores (gross motor function, fine motor function and visuospatial coordination, language and sociability) in children aged 2-30 months and calculates an overall neurodevelopmental score with a mean of 100 , and an SD of 15 . The number of infants with cerebral palsy, deafness, blindness, developmental delay (neurodevelopmental score below 85 ) will be recorded in the same population during a paediatric consultation at 24 months corrected age.

\section{Psychological outcomes}

\section{Mothers}

Various mental health symptoms of the mothers will be assessed using several validated self-report questionnaires. The questionnaires will be completed by mothers reading French fluently. i. Depressive symptoms in the last 7 days will be measured 15 days after birth and at 6 months corrected age using the Edinburgh Postnatal Depression Scale (EPDS) ${ }^{35}$ which has been validated for pregnant women $^{36}$ and in a French sample, with good psychometric properties. ${ }^{37}$

ii. Anxiety symptoms will be assessed 15 days after birth and at 6 months corrected age using the Hospital Anxiety and Depression Scale, validated in French, with good psychometric properties. ${ }^{38-40}$ The anxiety subscale has seven items measuring state anxiety in the last 7 days. It may be used as a measure of symptom severity.

iii. Mother-infant bonding will be measured using the Mother-Infant Bonding Scale, ${ }^{41} 42$ days after birth and at 6 months corrected age. In this questionnaire, the mother rates eight adjectives describing her feelings towards her infant that are indicative of motherinfant bonding. ${ }^{41} 42$ This questionnaire was translated into French. ${ }^{43}$

iv. Maternal post-traumatic stress disorder (PTSD) symptoms will be assessed 1 month after childbirth and at 6 months corrected age using the Post-traumatic Checklist for the Diagnostic and Statistical Manual of Mental Disorders, Fifth Edition (DSM-5). ${ }^{44}$ This questionnaire has 20 items, measuring the 20 DSM5 symptoms of PTSD. It was translated into French and can also be scored to provide a provisional PTSD diagnosis.

v. Parenting stress will be evaluated at 6 months corrected age using the French version of the Parenting Stress Index Fourth Edition Short Form (PSI), ${ }^{46}$ which has 36 items assessing parental distress, dysfunctional parent-child interactions and child difficulties. The PSI has good psychometric properties. ${ }^{47}$

Satisfaction of the mothers regarding the breastfeeding support and the intervention will also be assessed with a questionnaire designed for the study.

\section{Infant behaviours}

Infant behaviour will be measured at 6 months corrected age using the French version of the Infant Behaviour Questionnaire-Revised Very Short Form. ${ }^{48}$ The mother 
reports on a 7-point Likert scale the frequency of her infant's behaviours during the previous 2 weeks. ${ }^{48}$

\section{Peer counsellors}

Depressive symptoms will be measured with the EPDS..$^{35} 37$

The satisfaction of peer counsellors will be measured using a questionnaire designed for the study.

\section{Partners}

The partner's satisfaction on the intervention will be evaluated with a questionnaire designed for the study.

\section{Economic outcomes}

Cost data during the first 2 years will be gathered from a subpopulation of infants included by centres in the Rhone-Alps region. To assess the total cost of each group, the amount of resources consumed (eg, consultations, hospitalisation, drugs, medical devices) will be extracted from the regional healthcare database and completed by the parents with their additional expenses (not covered by 'the national social security system').

The primary economic endpoint will be the incremental cost-effectiveness ratio (ICER) at 2 years corrected age for intervention group versus control group. It will be expressed as incremental cost per impairment avoided. This outcome will be measured in the same subgroup as the neurodevelopmental outcome.

The secondary economic endpoint will be the costconsequence analysis at 2 years corrected age. The health outcomes considered will be the mortality and the hospitalisation rates.

\section{Feasibility of the intervention}

The implementation of the intervention will be reported: number and characteristics of counsellors (age, place of birth, study level, parity, age of the previous preterm child), number of face-to-face meetings and of videotelephony or telephone contacts, duration and subjects treated in the meetings.

The number of mothers declining peer support counselling will be collected with the reason for refusal. The duration of counselling and the proportion of mothers ending the peer counselling prematurely and their reasons will also be collected.

\section{Healthcare satisfaction}

The satisfaction and the acceptability of the intervention by healthcare professionals (physicians and nurses) will also be evaluated by the specific questionnaires developed for the study.

\section{Blinding}

Owing to the nature of the intervention, healthcare providers, parents and researchers will not be blind during the intervention phase.

\section{Other changes occurring in the NICU during the study period}

All events, such as organisational modifications that occur during the study period and may interact with the intervention or the study results will be recorded in a logbook.

\section{Study sample size}

We calculated the sample size for the stepped-wedge trial using the method reported by Hussey and Hughes. ${ }^{49}$ The breastfeeding rate at 2 months corrected age was $15 \%$ according to French regional data (Réseau Ecl'aur, 2017). We expected a relative improvement of $50 \%$ in the primary outcome, that is, an increase from $15 \%$ in the control group to $22.5 \%$ in the group with intervention. The coefficient of variation was set at 0.1 for a compromise between the recruitment capability of the sites and the required power. The type I error was fixed at $5 \%$ for a bilateral test. Under these hypotheses, the inclusion of 1800 mother-child dyads will allow to reach an approximate power of $80 \%$. To account for lost to follow-up and refusal of the intervention, we have added another $15 \%$ providing a sample of 2080 mother-child dyads. Knowing that when possible, all mother-infant pairs will be included in the case of a multiple birth and assuming a $15 \%$ rate of plural births, a total of 2400 mother-child dyads should be enrolled.

\section{Analysis}

General analysis principles

A full statistical analysis plan will be finalised prior to database lock. Statistical analysis and results will be reported according to the Consolidated Standards of Reporting Trials (CONSORT) guidelines for stepped wedge cluster randomised controlled trial. ${ }^{5051}$

The analysis will be performed according to the intention-to-treat principle. The individual centres will be the unit of randomisation and the individual motherchild dyads will be the unit of analysis. ${ }^{52}$ All statistical tests and CIs will be two sided with a type I error set at alpha $=0.05$.

\section{Descriptive analysis}

Descriptive statistics will be calculated and compared with baseline characteristics of mother-child dyads enrolled during the control periods and the intervention periods using the $\chi^{2}$ test for categorical variables and the Student's t-tests for quantitative variables. ${ }^{53}$ Descriptive statistics will be provided at the individual and cluster level using the aggregate summary data.

\section{Analysis of the primary outcomes}

To compare the breastfeeding rate at 2 months corrected age, we will use generalised linear mixed models with a random effect for cluster, a fixed effect indicating the group assignment of each cluster at each step and a fixed effect of time (each period) to account for potential secular changes during the study period. ${ }^{495455}$ The underlying form of time will be included in the model as a linear term or polynomial term, as appropriate. Further models will be fitted to test the heterogeneity of intervention across centres (including an interaction between intervention and centre as a fixed effect) and to 
test the heterogeneity of intervention across time periods (including an interaction between intervention and periods as a fixed effect). Analyses will also be adjusted for cluster-level covariates and for individual-level covariates unbalanced at baseline or known to be associated with breastfeeding status, such as mother's ethnicity, smoking status, mother's education level, breastfeeding history, caesarean delivery, gestational age, multiple birth and recorded differences in usual cares. Duration of peer support will also be controlled for. Results will be expressed as OR and $95 \%$ CIs.

The breastfeeding rate at 2 months corrected age will be likely to be censored because of death and lost to follow-up. If so, the impact of intervention will be assessed using a Cox proportional hazard regression models. The estimated intervention effect will be reported as HR with $95 \%$ CIs.

\section{Implementation}

Descriptive statistical analysis will be performed on data relating to peer counsellors (number of peer counsellors recruited per centre, sociodemographic characteristics), on process variables to describe the intervention implementation in each centre (number of contacts received by dyad from peer counsellor, duration of contacts received, number of mothers who refused the intervention, number of mothers who interrupted the intervention and reason) and, on data related to satisfaction.

\section{Analysis of the secondary outcomes}

Analysis of the secondary outcomes will be conducted in the same way as for the primary outcome. Logistic regressions will be used for binary measurements, linear regression models for continuous measurements and Cox proportional hazard regression models for survival analysis. The secondary outcomes will be exploratory.

\section{Economic evaluation}

For both economic endpoints, costs will be evaluated from a societal perspective. The French healthcare tariffs will be used to cost out resources consumed during the follow-up period.

The specific cost of the intervention (training time, indemnity for counsellors and extracosts due to an increased working time of the referent nurses or lactation consultants) will be included. Each component will be costed out using a unit production cost or a purchasing price.

The ICER will be defined by the difference in cost between the two interventions, divided by the difference in outcome expressed as the number of infants without impairment in each group.

The health outcomes of the cost-consequence analysis will be presented separately. Moreover, a budget impact analysis will be performed.

\section{Sensitivity analysis}

Breastfeeding outcomes will be reanalysed in several post hoc sensitivity and restricted analyses. First, we will perform the analysis in the subgroup of mother-child dyads with a minimum intervention duration of 6 weeks. Second, the Swiss mother-child dyads will be excluded from the analysis, as a light version of peer counsellor support is already proposed in Switzerland.

The ICER will be calculated and sensitivity analysis (deterministic and probabilistic) will be performed to address uncertainty in cost and outcomes across both groups.

\section{Missing data}

There will be no imputation of missing data. Every effort will be made to minimise missing data including during a follow-up.

\section{Adverse events}

Unexpected serious adverse events will be reported within 48 hours to the primary investigator of the study and to the members of the Data Safety and Monitoring Board in Switzerland. In France, due to the low risks, the adverse event declaration will follow the standard procedure of each hospital.

\section{Data management}

All study data will be entered by research staff in an electronic CRF. All data will be precoded and stored in a secured database.

\section{Monitoring}

Monitoring will be performed by a qualified person independent of the study group. Monitoring will check the notification of participation in the study and of no-opposition of the parents in a sample of 20 participants in each centre. Specific consent will also be checked for participants in the subgroup used for the economic study. At each visit, a CRF will be checked for eligibility criteria and the main outcome measure. If any deviation is noted, an additional monitoring visit will be performed.

\section{ETHICS AND DISSEMINATION}

The study will be conducted in accordance with the 1964 Declaration of Helsinki and its later amendments. Informed consent will be obtained by investigators from all individual participants involved in the study, this consent is oral in France and written in Switzerland and Belgium. A specific written consent is obtained for the ancillary economic study. Important protocol modifications will be communicated to relevant parties following the relevant procedures.

All stored data are anonymised and protected by a password. The identification data are stored independently in another computer with another password.

The sponsor has an insurance to cover any harm from trial participation.

The study team will be committed to full disclosure of the results of the trial. The results of the study will be disseminated at several national and international academic and clinical meetings, and as articles published in national 
and international peer-reviewed journals. The study will be implemented and reported in-line with the CONSORT statement. Each paper or abstract will be submitted to the appropriate subcommittee for review of its appropriateness and scientific merit prior to submission. The study team will adhere to defined authorship criteria as per the International Committee of Medical Journal Editors. We used the Standard Protocol Items: Recommendations for Interventional Trials reporting guidelines for the present publication. $^{56}$

\section{Patient and public involvement}

The design of the study was submitted to the 'Réseau d'information et de soutien à l'allaitement maternel' (breastfeeding information and support's network) in which an association of breastfeeding mothers is implicated. Furthermore, our intervention is carried out by peers (experts by experience).

\section{SIGNIFICANCE AND OUTLOOK}

Although the mother's own milk is an important protector in preterm infants, breast feeding remains more difficult, less frequent and shorter in this vulnerable population. There is thus a critical need of evidence-based strategies to enhance breastfeeding outcomes in the NICU and after discharge. This large multicentre study provides one of the first opportunities to test the feasibility and efficacy of breastfeeding peer counselling support system in Western European countries. Similarly to what has been shown for term infants in different settings, breastfeeding peer support could indeed constitute a feasible, acceptable, effective, efficient and thus sustainable intervention for preterm neonates. The provided emotional support could potentiate existing professional breastfeeding support.

We will evaluate the efficacy of the intervention on breastfeeding outcomes, neonatal mortality, severe morbidities, neurodevelopment and psychological health of the mother and mother-infant bounding, as well as parenting stress. Due to the nature of the intervention, the communication with the mother is key, and sufficient French language skills are thus necessary. This will likely limit the inclusion of low-income families. Psychological outcomes will solely be measured by self-report questionnaires, which is another limitation of the study. It would have been interesting to include a qualitative process evaluation component.

Finally, the economic evaluation is based on infants from a specific French geographical region only. The results will, therefore, not be generalisable to the entire population. It was not feasible with the funding obtained to study a larger population. However, if the study demonstrates that a peer counselling support is an effective and cost-efficient strategy to support and improve breast feeding in NICUs, the intervention is likely to be widely implemented.

\section{Author affiliations}

${ }^{1}$ Hopital Femme Mère Enfant, Neonatology, Hospices Civils de Lyon, Bron, France

${ }^{2}$ Service de Biostatistique-Bioinformatique, Pôle Santé Publique, Hospices Civils de Lyon, Lyon, France 3équipe Biostatistique-Santé, Laboratoire de Biométrie et Biologie Évolutive, CNRS UMR 5558, Villeurbanne, France

${ }^{4}$ Institute of Higher Education and Research in Healthcare, University of Lausanne, Lausanne, Switzerland

${ }^{5}$ Woman-Mother-Child, Lausanne University Hospital, Lausanne, Switzerland

${ }^{6}$ Pôle Santé Publique, Hospices Civils de Lyon, Lyon, France

${ }^{7}$ Laboratoire Health Services and Performance Research, EA 7425 HESPER, Université Lyon 1, Villeurbanne, France

${ }^{8}$ Public Health, University Hospital Centre Lyon, Lyon, France

${ }^{9}$ Child and Adolescent Psychiatry, Centre Hospitalier Universitaire Vaudois, Lausanne, Vaud, Switzerland

${ }^{10}$ Hopital Femme Mère Enfant, Neonatology, Hospices Civils de Lyon, Bron, Auvergne-Rhône-Alpes, France

${ }^{11}$ Equipe P2S4129, Université Claude Bernard Lyon 1, Villeurbanne, AuvergneRhône-Alpes, France

${ }^{12}$ Pôle Santé Publique, Hospices Civils de Lyon, Lyon, France

${ }^{13}$ Laboratoire Health Services and Performance Research (HESPER) EA 7425,

Université de Lyon 1, Villeurbanne, France

Acknowledgements We thank Danièle Bruguière for help with the conception of the intervention, Thi-Huyen-Trang Nong and Marie Josée Communal for help with conception of the study, the members of the 'Réseau d'Information et Soutien à l'Allaitement Maternel' for their advice on the design of the intervention, Christell Julien for her implication in the design of the economic evaluation.

Contributors SL chose the subject, wrote the initial draft; ST proposed the design; PO participated in the design and the redaction; JM designed the medico economic part; $\mathrm{AD}$ designed the statistical analysis; $\mathrm{AH}$ and $\mathrm{MMH}$ designed the part on mental health; OC and CJFF contributed to plan the study. All the authors reviewed and revised the manuscript, and approved the final manuscript as submitted.

Funding This study is supported by a grant from the Programme de Recherche sur la Performance du Système de soins (PREPS 16-0373) from the French Ministry of Health (Ministère chargé de la Santé, Direction de l'Hospitalisation et de l'Organisation des Soins) and a grant from Fondation Planètes Enfants Malades and, Fondation Lotty Buol.

Competing interests None declared.

Patient consent for publication Not required.

Ethics approval The protocol has obtained the ethics approval from the 'Comite de Protection des Personnes Sud-Est VI' with the ID-RCB: 2017-A01977-46 in France. Local ethic committees have approved the study in Belgium (Comité d'Ethique du CHVE) and in Switzerland (Commission cantonale d'éthique de la recherche sur l'être humain).

Provenance and peer review Not commissioned; externally peer reviewed.

Open access This is an open access article distributed in accordance with the Creative Commons Attribution Non Commercial (CC BY-NC 4.0) license, which permits others to distribute, remix, adapt, build upon this work non-commercially, and license their derivative works on different terms, provided the original work is properly cited, appropriate credit is given, any changes made indicated, and the use is non-commercial. See: http://creativecommons.org/licenses/by-nc/4.0/.

ORCID iDs

Sophie Laborie http://orcid.org/0000-0002-3257-6050

Jennifer Margier http://orcid.org/0000-0003-2590-4228

\section{REFERENCES}

1 Victora CG, Bahl R, Barros AJD, et al. Breastfeeding in the 21st century: epidemiology, mechanisms, and lifelong effect. Lancet 2016;387:475-90.

2 Rollins NC, Bhandari N, Hajeebhoy N, et al. Why invest, and what it will take to improve breastfeeding practices? Lancet 2016;387:491-504.

3 Schanler RJ, Shulman RJ, Lau C. Feeding strategies for premature infants: beneficial outcomes of feeding fortified human milk versus preterm formula. Pediatrics 1999;103:1150-7.

4 Patel AL, Johnson TJ, Engstrom JL, et al. Impact of early human milk on sepsis and health-care costs in very low birth weight infants. $J$ Perinatol 2013;33:514-9.

5 Hylander MA, Strobino DM, Dhanireddy R. Human milk feedings and infection among very low birth weight infants. Pediatrics 1998;102:E38. 
6 Corpeleijn WE, Kouwenhoven SMP, Paap MC, et al. Intake of Own Mother's Milk during the First Days of Life Is Associated with Decreased Morbidity and Mortality in Very Low Birth Weight Infants during the First 60 Days of Life. Neonatology 2012;102:276-81.

7 Lucas A, Cole TJ. Breast milk and neonatal necrotising enterocolitis. Lancet 1990;336:1519-23.

8 Hylander MA, Strobino DM, Pezzullo JC, et al. Association of human milk feedings with a reduction in retinopathy of prematurity among very low birthweight infants. J Perinatol 2001;21:356-62.

9 Spiegler J, Preuß M, Gebauer C, et al. Does Breastmilk Influence the Development of Bronchopulmonary Dysplasia? J Pediatr 2016;169:76-80.

10 Elder DE, Hagan R, Evans SF, et al. Hospital admissions in the first year of life in very preterm infants. $J$ Paediatr Child Health 1999:35:145-50.

11 Johnson TJ, Patra K, Greene MM, et al. Nicu human milk dose and health care use after NICU discharge in very low birth weight infants. $J$ Perinatol 2019;39:120-8.

12 Vohr BR, Poindexter BB, Dusick AM, et al. Persistent beneficial effects of breast milk ingested in the neonatal intensive care unit on outcomes of extremely low birth weight infants at 30 months of age. Pediatrics 2007;120:e953-9.

13 Rozé J-C, Darmaun D, Boquien C-Y, et al. The apparent breastfeeding paradox in very preterm infants: relationship between breast feeding, early weight gain and neurodevelopment based on results from two cohorts, EPIPAGE and lift. BMJ Open 2012;2:e000834.

14 Gibertoni D, Corvaglia L, Vandini S, et al. Positive effect of human milk feeding during NICU hospitalization on 24 month neurodevelopment of very low birth weight infants: an Italian cohort study. PLoS One 2015;10:e0116552.

15 Lucas A, Morley R, Cole TJ, et al. Breast milk and subsequent intelligence quotient in children born preterm. Lancet 1992;339:261-4.

16 Patra K, Hamilton M, Johnson TJ, et al. Nicu human milk dose and 20-Month neurodevelopmental outcome in very low birth weight infants. Neonatology 2017;112:330-6.

17 Bonet M, Blondel B, Agostino R, et al. Variations in breastfeeding rates for very preterm infants between regions and neonatal units in Europe: results from the mosaic cohort. Arch Dis Child Fetal Neonatal Ed 2011;96:F450-2.

18 Jolly K, Ingram L, Khan KS, et al. Systematic review of peer support for breastfeeding continuation: metaregression analysis of the effect of setting, intensity, and timing. BMJ 2011;344:d8287.

19 Sudfeld CR, Fawzi WW, Lahariya C. Peer support and exclusive breastfeeding duration in low and middle-income countries: a systematic review and meta-analysis. PLoS One 2012;7:e45143.

20 Agrasada GV, Gustafsson J, Kylberg E, et al. Postnatal peer counselling on exclusive breastfeeding of low-birthweight infants: a randomized, controlled trial. Acta Paediatr 2005;94:1109-15.

21 Shakya P, Kunieda MK, Koyama M, et al. Effectiveness of community-based peer support for mothers to improve their breastfeeding practices: a systematic review and meta-analysis. PLoS One 2017;12:e0177434.

22 Trickey H, Thomson G, Grant A, et al. A realist review of one-to-one breastfeeding peer support experiments conducted in developed country settings. Matern Child Nutr 2018;14:e12559.

23 Preyde M, Ardal F. Effectiveness of a parent "buddy" program for mothers of very preterm infants in a neonatal intensive care unit. CMAJ 2003;168:969-73.

24 Merewood A, Chamberlain LB, Cook JT, et al. The effect of peer counselors on breastfeeding rates in the neonatal intensive care unit: results of a randomized controlled trial. Arch Pediatr Adolesc Med 2006;160:681-5.

25 Oza-Frank R, Bhatia A, Smith C. Combined peer counselor and lactation consultant support increases breastfeeding in the NICU. Breastfeeding Medicine 2013;8:509-10.

26 Kaunonen M, Hannula L, Tarkka M-T. A systematic review of peer support interventions for breastfeeding. J Clin Nurs 2012;21:1943-54.

27 Rogers C. La relation d'aide et la psychothérapie, Paris, Edition ESF, 2008.

28 Nyqvist KH, Häggkvist A-P, Hansen MN, et al. Expansion of the Baby-Friendly Hospital initiative ten steps to successful breastfeeding into neonatal intensive care. J Hum Lact 2013;29:300-9.

29 Papile L-A, Burstein J, Burstein R, et al. Incidence and evolution of subependymal and intraventricular hemorrhage: a study of infants with birth weights less than 1,500 GM. J Pediatr 1978;92:529-34.
30 Bell MJ, Ternberg JL, Feigin RD, et al. Neonatal necrotizing enterocolitis. therapeutic decisions based upon clinical staging. Ann Surg 1978;187:1-7.

31 Isayama T, Lee SK, Yang J, et al. Revisiting the definition of bronchopulmonary dysplasia: effect of changing Panoply of respiratory support for preterm neonates. JAMA Pediatr 2017:171:271-9.

32 International Committee for the classification of retinopathy of prematurity. The International classification of retinopathy of prematurity revisited. Arch Ophthalmol 2005;123:991-9.

33 Stoll BJ, Hansen N, Fanaroff AA, et al. Late-Onset sepsis in very low birth weight neonates: the experience of the NICHD neonatal research network. Pediatrics 2002;110:285-91.

34 Josse D. Brunet-Lezine révisé: Echelle de développement psychomoteur de la première enfance. Paris: Etablissements d'applications psychotechniques, 1997.

35 Cox JL, Holden JM, Sagovsky R. Detection of postnatal depression. development of the 10-item Edinburgh postnatal depression scale. Br J Psychiatry 1987;150:782-6.

36 Bunevicius A, Kusminskas L, Pop VJ, et al. Screening for antenatal depression with the Edinburgh depression scale. Journal of Psychosomatic Obstetrics \& Gynecology 2009;30:238-43.

37 Guedeney N, Fermanian J. Validation study of the French version of the Edinburgh postnatal depression scale (EPDS): new results about use and psychometric properties. European Psychiatry 1998;13:83-9.

38 Bocéréan $\mathrm{C}$, Dupret $\mathrm{E}$. A validation study of the hospital anxiety and depression scale (HADS) in a large sample of French employees. BMC Psychiatry 2014;14:354.

39 Herrmann C, Buss U, Snaith R. Hospital Anxiety and Depression Scale-Deutsche Version [Ein Fragebogen von Angst und Depressivität in der somatischen Medizin. Bern, Schweiz: Hans Huber, 1995.

40 Zigmond AS, Snaith RP. The hospital anxiety and depression scale. Acta Psychiatr Scand 1983;67:361-70.

41 Taylor A, Atkins R, Kumar R, et al. A new mother-to-infant bonding scale: links with early maternal mood. Arch Womens Ment Health 2005;8:45-51.

42 van Bussel JCH, Spitz B, Demyttenaere K. Three self-report questionnaires of the early mother-to-infant bond: reliability and validity of the Dutch version of the MPAS, PBQ and MIBS. Arch Womens Ment Health 2010;13:373-84.

43 Horsch A, Jacobs I, Gilbert L, et al. Impact of perinatal asphyxia on parental mental health and bonding with the infant: a questionnaire survey of Swiss parents. Bmjpo 2017;1:e000059.

44 Weathers F, Litz B, Herman D, et al. The PTSD checklist (PCL): reliability, validity, and diagnostic utility. Paper presented at the Annual Meeting of the International Society for Traumatic Stress Studies, 1993.

45 Weathers FW, Litz BT, Keane TM, et al. The PTSD checklist for DSM5 (PCL-5). National Center for PTSD, 2013.

46 Abidin R. Parenting stress index ((psi). Odessa, FL: Psychological Assessment Resources. In: Inc, 1995.

47 Singer LTet al. Maternal psychological distress and parenting stress after the birth of a very low-birth-weight infant. JAMA 1999;281:799-805.

48 Putnam SP, Helbig AL, Gartstein MA, et al. Development and assessment of short and very short forms of the infant behavior Questionnaire-Revised. J Pers Assess 2014;96:445-58.

49 Hussey MA, Hughes JP. Design and analysis of stepped wedge cluster randomized trials. Contemp Clin Trials 2007;28:182-91.

50 Campbell MK, Piaggio G, Elbourne DR, et al. Consort 2010 statement: extension to cluster randomised trials. BMJ 2012;345:e5661.

51 Hemming K, Haines TP, Chilton PJ, et al. The stepped wedge cluster randomised trial: rationale, design, analysis, and reporting. BMJ 2015;350:h391.

52 Davey C, Hargreaves J, Thompson JA, et al. Analysis and reporting of stepped wedge randomised controlled trials: synthesis and critical appraisal of published studies, 2010 to 2014. Trials 2015;16:358.

53 Bolzern JE, Mitchell A, Torgerson DJ. Baseline testing in cluster randomised controlled trials: should this be done? BMC Med Res Methodol 2019;19:106.

54 Hemming K, Taljaard M, Forbes A. Analysis of cluster randomised stepped wedge trials with repeated cross-sectional samples. Trials 2017;18:101.

55 Thompson JA, Fielding KL, Davey C, et al. Bias and inference from misspecified mixed-effect models in stepped wedge trial analysis. Stat Med 2017:36:3670-82. 
